\title{
Ultrasound and microbubbles for treatment purposes: mechanisms and results
}

\author{
F. Tranquart $\cdot$ T. Bettinger $\cdot$ J. M. Hyvelin
}

Received: 7 October 2013/Accepted: 30 January 2014/Published online: 21 February 2014

(C) Italian Association of Nuclear Medicine and Molecular Imaging 2014

\begin{abstract}
The need to improve local drug and gene delivery, to increase their efficacy and safety, has prompted scientists to investigate different approaches. One of the most promising has been the combination of ultrasound and microbubbles, which can act as a local enhancer of this delivery. Even though the exact mechanism has not been fully elucidated, close contact between the bubbles and cell membrane is required to induce pore formation or endocytosis prior to secondary transfer of the molecule of interest into the cell. The literature contains a wealth of papers that have clearly demonstrated the efficiency of this approach in animal models. The clinical effectiveness of microbubble-mediated treatment demonstrated in sonothrombolysis will likely open up new treatment opportunities in refractory diseases or the possibility of developing new means of delivering molecules in poorly accessible tissues, such as brain tissue.
\end{abstract}

Keywords Sonoporation · Drug delivery · Gene delivery $\cdot$ Ultrasound $\cdot$ Sonothrombolysis

\section{Introduction}

The extensive use of chemotherapy in oncology has led to considerably improved life expectancy of cancer patients. In many cases, however, achievement of this objective is hampered by the occurrence of adverse events which can

Color figures online at http://link.springer.com/article/10.1007/ s40336-014-0052-4

F. Tranquart $(\bowtie) \cdot$ T. Bettinger $\cdot$ J. M. Hyvelin

Geneva Research Center and Manufacturing Site, Bracco Suisse

SA, Route de la Galaise, 31, 1228 Plan les Ouates, Switzerland

e-mail: francois.tranquart@bracco.com lead to suspension or lowering of the dose and thus to secondary reduced efficacy. For this reason, researchers around the world have endeavoured to propose alternative solutions capable of increasing the presence of the drug at the desired location while reducing secondary adverse events. In view of the inherently toxic nature of the drugs used in oncology to reduce the tumoral mass, reports of adverse events are, in some cases, considered a sign of efficacy. However, in most cases, adverse events are the consequence of the excessively high circulating drug concentrations needed to achieve efficacy at the tumour site. Several options might be pursued to resolve this issue: (1) identification of a drug that can accumulate at the tumour site, (2) identification of a pharmaceutical means of improving the tumour/healthy tissue ratio, or (3) use of an additional method to increase the tumour accumulation/ delivery of the drug in relation to its circulatory level [15].

With regard to the latter proposal, ultrasound appears to be one of the most attractive external trigger approaches for improving/enhancing drug delivery to the tumour site. The possibility of tuning ultrasound settings to allow delivery of acoustic pressure with various amplitudes at the desired site has been reported for decades. Thanks to the improvements made to ultrasound scanners over recent years, today's clinical scanners could offer this opportunity and play a role in drug/gene delivery. It has, however, been shown that the local presence of microbubbles, potentiating the efficiency of ultrasound waves, is necessary to make them really effective for this purpose [34].

Indeed, it is generally acknowledged that gas-filled microbubbles reinforce the effect of ultrasound waves and thus allow greater delivery efficiency than is possible in the absence of microbubbles. This combination, a technique commonly known as sonoporation, has been proposed to improve the local delivery of drugs, mostly anticancer 
drugs and nucleic acids, with the aim of increasing efficacy and/or decreasing adverse events. This approach has also been used for accelerating clot dissolution in stroke patients, although the underlying mechanism of sonothrombolysis remains to be fully elucidated.

The purpose of the present paper is to report the proposed mechanisms of action of sonoporation at the local site and some of the most clinically relevant results from the literature.

\section{Description of microbubbles}

The microbubbles currently approved for clinical use or under development in various laboratories contain fluorinated gases, and are stabilised using a shell and several surfactants. These two factors (the low diffusion gas and the combination of shell plus surfactants) have been shown to increase the persistence of the microbubbles to up to several minutes within blood circulation. To avoid lung retention, microbubbles are typically less than $3 \mu \mathrm{m}$ in diameter and are therefore smaller than red blood cells [33]. Microbubbles can be administered intravenously or into certain cavities, following drug administration (a bolus injection or continuous infusion using a pump when a steady-state concentration is required). Microbubbles were first developed to visualise/differentiate microcirculation from its surroundings. They act as strong ultrasound reflectors: under ultrasound waves, microbubbles oscillate and produce specific signals which are used for imaging. Used in the therapeutic setting, the interaction between ultrasound and microbubbles shows some specific features which will be addressed below [1]. It is important to note that microbubbles, purely because of their size, can only enter the blood compartment. Thus, microbubbles are considered a pure blood pool agent, simply travelling through the microvasculature without the possibility of reaching the interstitial compartment.

\section{Clinically approved microbubbles}

SonoVue ${ }^{\circledR}$ (Bracco, Milan, Italy) is approved for radiology and cardiac indications in Europe and other countries in Asia. SonoVue ${ }^{\circledR}$ is a lipid-shelled contrast agent based on microbubbles of a fluorinated gas (sulphur hexafluoride, $\mathrm{SF}_{6}$ ). This agent is stored as a lyophilisate and is ready for injection after resuspension with saline [8].

Definity ${ }^{\circledR}$ (Lantheus, USA) is approved in the USA for cardiac applications. It is a suspension of phospholipidencapsulated perfluoropropane $\left(\mathrm{C}_{3} \mathrm{~F}_{8}\right)$ microbubbles that requires storage at low temperature and activation by mechanical shaking before injection [8].

Optison $^{\circledR}$ (Mallinckrodt, Germany) is approved for cardiac indication in Europe and USA. The microbubbles are obtained by sonification of human albumin and perfluoropropane $\left(\mathrm{C}_{3} \mathrm{~F}_{8}\right)$ [8].

Sonazoid $^{\circledR}$ (GE Healthcare, USA) consists of perfluorobutane $\left(\mathrm{C}_{4} \mathrm{~F}_{10}, \mathrm{PFB}\right)$ microbubbles stabilized by a monomolecular membrane of hydrogenated egg phosphatidyl serine, embedded in an amorphous sucrose structure. It is approved for radiology and breast indications in Japan [8].

Modified microbubbles

As microbubbles, due to their size, are unable to reach the interstitial compartment, the use of nanodroplets, which are smaller than microbubbles but otherwise rather similar to them, has been proposed. These nanodroplets could extravasate more easily than microbubbles and reach the interstitial compartment. The perfluorocarbon gas used in this context is in liquid form at room temperature, while a phase transition under acoustic activation vaporises the gas leading to the secondary appearance of a bubble which can oscillate according to acoustic waves [29].

Although the most common use of microbubbles for sonoporation relied on separate injections of free microbubbles and drug under different formulations, ranging from micelles to liposomes, it has been suggested that the drug could be carried directly on the bubble itself, thereby improving its local delivery [17]. To this end, various methods have been tested, considering the size of the bubble and its shell, the size of the drug, and the nature (hydrophilic or hydrophobic) of the drug. The molecule might be simply attached to the external side of the shell, inserted within the shell (necessitating a thicker shell than usual with the incorporation of oil or polymers as an example), or entrapped within the core of the microbubble (Fig. 1) [19]. The destruction of the microbubble upon its exposure to ultrasound exposure results in fragmentation of the oil and lipid shell, and the formation of small particles able to act as the drug carrier.

More complex structures have recently been proposed involving attachment of liposomes to the external side of the shell [21] (Fig. 2). This has been found to lead to a significant increase in the amount of drug to be carried to the site of interest.

However, it is important to bear in mind the relatively limited amount of agents that can be carried by such microbubbles, This is a limitation inherent to their structure, which allows loading of the drug only in the outer layer, the core being composed of gas. Castle et al. [5] found that a 3-MDa plasmid DNA, if stretched out to its maximum diameter, would appear as a single point relative to a $3 \mu \mathrm{m}$ diameter bubble. If a circular plasmid of $48 \mathrm{~nm}$ were to lie as a completely flat ring on the surface of the bubble, there would be enough bubble surface area to accommodate 

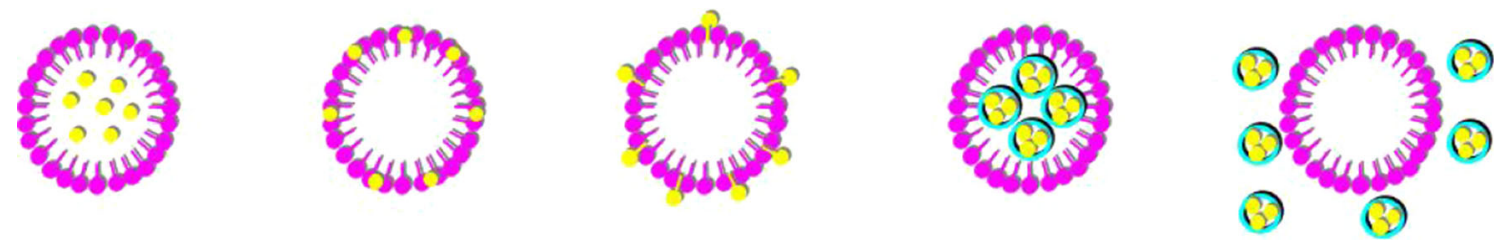

Fig. 1 Proposed mechanisms of drug loading within a bubble with a payload (in yellow) which can be within the core, inserted within the shell, attached to the shell, within the core or attached to the shell as nanoparticles (from left to right) (color figure online)

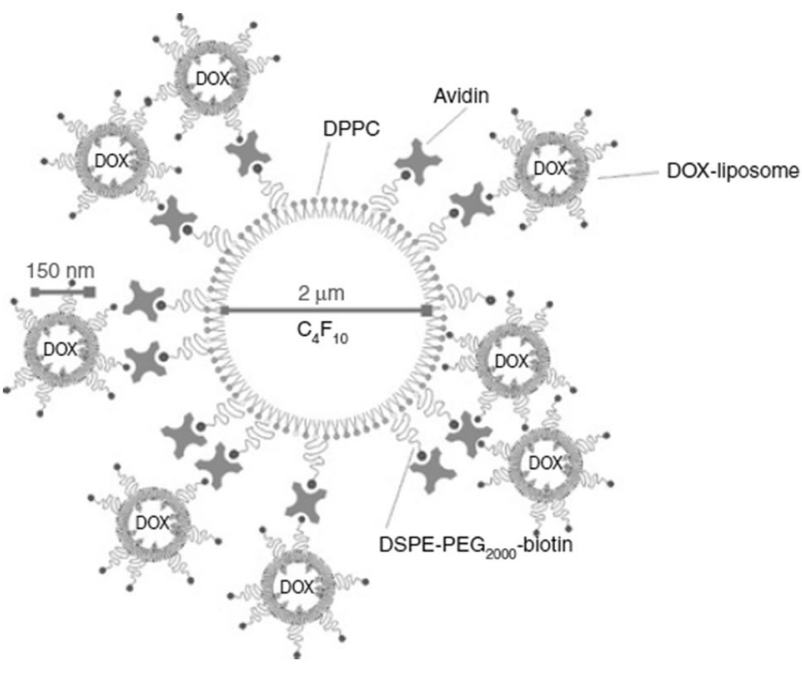

Fig. 2 Schematic presentation of a DOX-liposome-loaded microbubble (not in scale). Biotinylated DOX-containing liposomes were attached to the surface of a biotinylated lipid microbubble with the aid of an avidin molecule. The mean sizes of liposomes and microbubbles are indicated on the picture. C4F10, perfluorobutane; DOX, doxorubicin; DPPC, dipalmitoylphosphatidylcholine; DSPE-PEG2000-biotin, [(1,2-distearoyl-sn-glycero-3-phosphoethanolamine- $N$-(biotinyl (polyethyleneglycol)2000)] [21]

12,000 DNA molecules. Of course, plasmid DNA does not exist to any meaningful extent as a flat ring, which means that considering steric hindrance alone, there is room on a $3 \mu \mathrm{m}$ bubble for more than 12,000 DNA molecules. The capacity for carrying small-molecule drugs would be much higher than that.

\section{Acoustic parameters}

Ultrasound-transmitted sequences used to induce gene delivery may be summarised in the following parameters: the transmission centre frequency (f0), the acoustic pressure (AP), the pulse length (PL), the pulse repetition frequency (PRF) and the total insonation time (TIT). The transmission centre frequency used for in vivo gene delivery ranges from 0.3 to $14 \mathrm{MHz}$. The choice of frequency depends on the size, and thus the resonance frequency, of the microbubbles [11]. In most of the reported studies, optimal delivery was observed at $1 \mathrm{MHz}$ for all types of microbubbles. The acoustic pressure applied during insonation is normally expressed as $\mathrm{kPa}$ or $\mathrm{MPa}$ as this corresponds to the acoustic amplitude. This is the most accurate parameter for comparing the settings used by different laboratories. In accordance with the international recommendations, it is probably important to consider the intensity, expressed as $\mathrm{W} \mathrm{cm}$, if a clinical application is envisaged. The increasing use of commercial ultrasound scanners for sonoporation has extended the use of the mechanical index (MI, expressed as the ratio of the peak negative pressure in MPa to the square root of the frequency in $\mathrm{MHz}$ ) for expressing the acoustic settings [13].

A certain level of acoustic pressure is required to create stable or inertial cavitation, which potentiates interactions between microbubbles and cell membrane and induces a transmembrane passage of the molecules of interest. This level depends on the ultrasound frequency used, as higher frequencies require significantly higher energy for the same efficiency. A pressure in the range of $1 \mathrm{MPa}$ is usually required to promote sonoporation, but this has to be validated for each condition and for the cells used. The size of drug that can be delivered into cells upon sonoporation has been measured in vitro and found to be in the range of a few tens of a nanometer, with a maximum diameter of $75 \mathrm{~nm}$ [25]. In addition, the duration of the cell membrane poration is in the range of few milliseconds, but according to some authors could reach a few hours $[13,38]$. When the sonoporation rate increases with the acoustic pressure, the cell death rate might also increase. Thus, the ratio of 'treated cells' over 'dead cells' has to be considered the real marker of efficacy.

Insonation time is an important factor in drug/gene delivery by sonoporation. This is a very active area of research, with some laboratories claiming that the use of short pulses is more efficient than the use of long pulses. One key parameter, called the duty cycle (DC), denotes the amount of time ultrasound is applied. This is linked to the pulse length and the pulse repetition. Various combinations might be proposed in this regard, also bearing in mind the following considerations: the possibility of the device used being able to deliver the acoustic energy without deleterious effects on the transducer itself (possible heating of the transducer) and the need for new microbubbles to enter and fill the insonated space after the destruction of the previous 
ones. Indeed, when microbubbles are destroyed within the tissue vasculature, the pulsing interval should be long enough to allow new ones to enter into the vasculature and refresh the target zone [6]. Therefore, each experiment requires careful control of such parameters to ensure maximal efficiency. It is also necessary to consider the impact of the acoustic pressure used on these parameters, making the choice of each one highly critical.

\section{Mechanisms of sonoporation}

Even though various hypotheses have been formulated in recent years to explain the sonoporation phenomenon, some aspects are still open for discussion since there is no evidence favouring any one mechanism. Despite this limitation, we can nevertheless summarise the main elements currently accepted by the scientific community on the basis of the literature. To understand the basic underlying mechanism, it is important to consider two consecutive steps linked to the specific nature of microbubbles (particle extravasation and particle uptake). Indeed, since microbubbles remain strictly within the vascular compartment, and since the objective is to improve the local delivery of drugs and genes, the first pre-requisite to allow extravasation of the drug is a close interaction between the microbubble, the cell, and the drug of interest.

\section{Particle extravasation}

When submitted to ultrasound waves, the bubbles oscillate next to the endothelium inducing a permeabilisation of this cell layer [3, 20]. Some experiments have suggested that ultrasound-microbubble interaction induces a transient opening of the gap junctions between cells allowing a passage of the particle of interest from the lumen towards the interstitial space $[3,15,22]$. This phenomenon might be controlled by varying acoustic pressures as described above. Blood-brain barrier (BBB) opening constitutes one of the most important permeability-enhancing applications of microbubbles plus ultrasound. The BBB is impermeable to molecules with a molecular weight higher than $400 \mathrm{Da}$, which therefore cannot reach the brain interstitium. This property results from the tight junctions between endothelial cells and from their highly controlled transport system. Several studies demonstrate that combining ultrasound and microbubbles allows localised BBB opening. This opening does not last for more than few hours with a return to a complete normal status under controlled ultrasound conditions. Similarly, local extravasation has been successfully applied pre-clinically to tumours, various organs, and skeletal muscle [10]. The case of BBB opening is of particular interest as it is typically obtained with high-
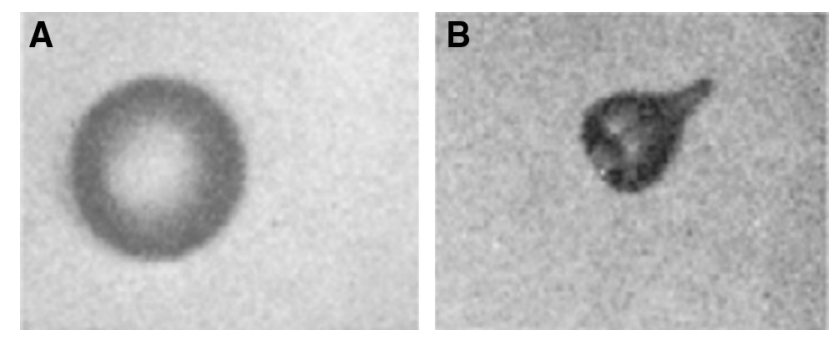

Fig. 3 US-induced jet formation in an experimental ultrasound contrast agent $(\mathrm{UCA})$ microbubble $(\mathrm{MI}=1.2)$. Each image frame corresponds to a $38 \times 30 \mu \mathrm{m}^{2}$ area. Interframe time is $0.33 \mu \mathrm{s}$ and exposure time is $10 \mathrm{~ns}$ [28]

amplitude ultrasound pulses and clear evidence has been obtained with non-invasive imaging techniques, allowing close monitoring of local efficiency [22]. In oncology, since the vessels are frequently leaky by essence, this increased vessel permeabilisation is of limited impact.

\section{Particle uptake}

The second step of drug/gene delivery is direct integration of the particle of interest within the targeted cell and it is a direct consequence of an increase in the permeability of the cell membrane. This phenomenon has been demonstrated by several groups and is reflected in an increase in ion fluxes as well as enhanced uptake of different small molecules (e.g. propidium iodide), macromolecules (e.g. fluorescent dextrans), or transgenes [13]. The underlying mechanism is still a matter of debate. Many possible hypotheses have been explored and demonstrated by different groups. It is likely that some of the proposed mechanisms are simultaneously involved in this increased cell membrane permeabilisation. These hypothesised mechanisms include lesions of the membrane by a direct physical effect due to the bursting bubble generating microstreaming, shock waves, and microjets [34]. In addition, a local increase in temperature [35] and the production of reactive oxygen species have been identified. It is to be noted that this cell membrane modification is transitory, since prolonged damage of the membrane would lead to cell death. This is a very important notion as it could be related to the treated cells/dead cells ratio, which is one the most important markers of efficacy of the selected method.

As described above, when the bubble bursts, the gas is expulsed from the bubble itself at very high velocities creating microstreaming and microjets [12, 28] (Fig. 3). These microjets may act as "micro needles", causing the formation of pores in cell membranes under optimal conditions. This phenomenon has been clearly identified and reported by Tachibana et al. [31]. During sonoporation, the shock waves physically create small transient ruptures in 
Fig. 4 Effects of the interaction of oscillating microbubbles on the cell membrane potential. a Video recordings of the interaction between microbubbles and the cell. In 1 , 3 , and 4 there is no ultrasound stimulation. In 2, 5, and 6 , the interaction between the bubble and the cell is weak. In 7 and 8 a direct contact is established.

b Membrane potential recordings. A transient interaction between membrane and UCA induced a transient hyperpolarization $(2,5$, and 6$)$. When the microbubble is strongly attached to the cell, a reproducible and synchronised hyperpolarisation is achieved (7 and 8 ) [30]
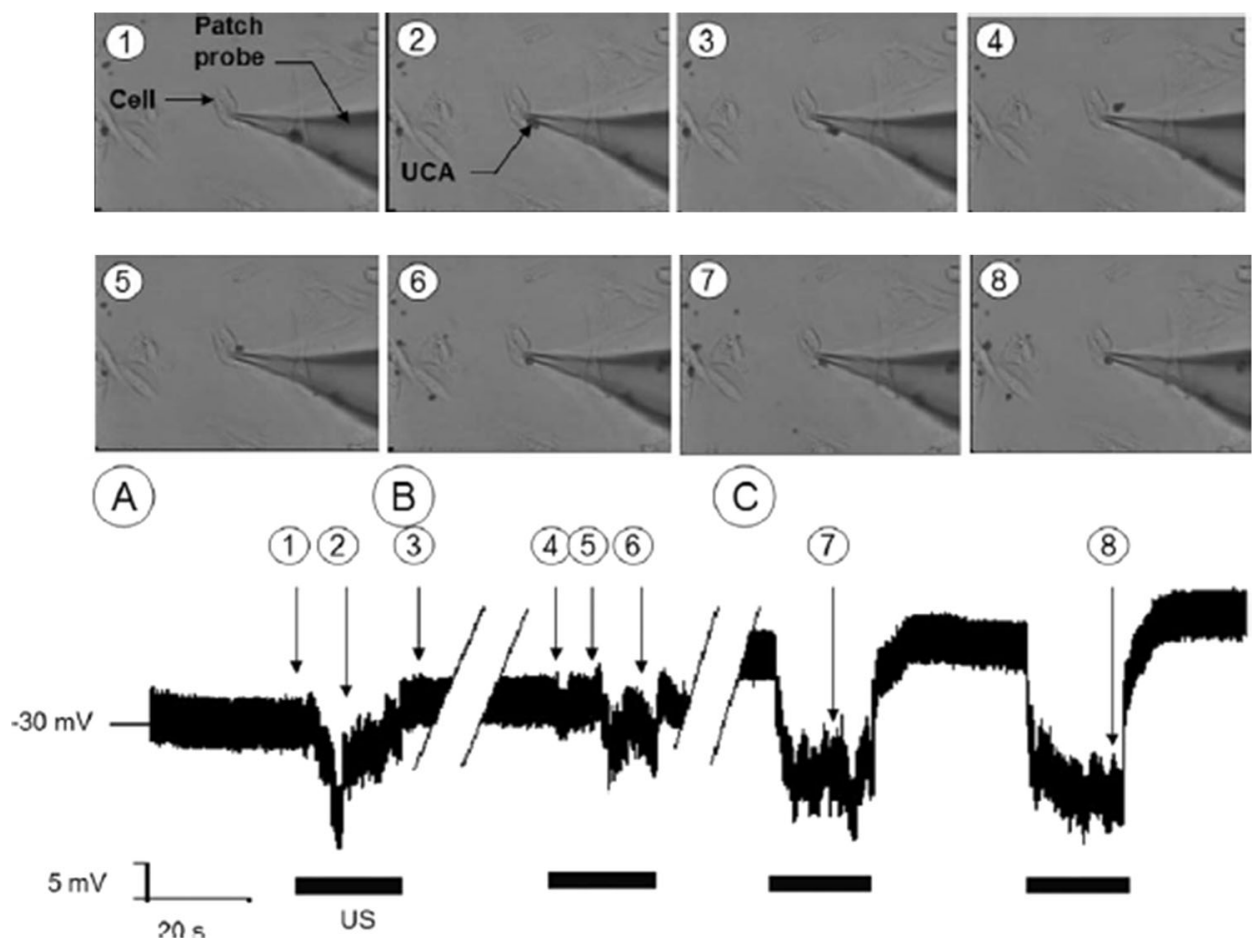

cell membranes in the order of $100 \mathrm{~nm}$ in diameter. They remain effectively open for up to $20 \mathrm{~s}$ allowing molecules and even DNA fragments to flow down their concentration gradient into the cell [25, 26, 37]. At higher acoustic pressures, most of the microbubbles can collapse asymmetrically particularly when there is only limited space for them to expand. Endocytosis and pores are now the most widely accepted consequences of these interactions, leading to the sonoporation phenomenon [26, 34, 39].

Once the particle of interest has crossed the cell membrane, it could reach various compartments of the cytoplasm and even the nucleus. Various direct observations have demonstrated the presence of fluorescence in the nucleus or the mitochondria, while the drug could remain free in the cytoplasm depending on its size [27].

There is some evidence to suggest that a low mechanical index (unlike a high one) could also play a role in this mechanism. Indeed, direct observation has shown that under acoustic activation, oscillation of the microbubbles next to the cell membrane could induce specific local changes that might allow transmembrane passage of the particle of interest without any cell membrane rupture [34]. Electrophysiological studies have clearly shown that bubble oscillation against the membrane, together with ultrasound activation, induces a cascade of physiological events characterised by membrane hyperpolarisation triggered by the activation of BKCa channels followed by an increase in intracellular $\mathrm{Ca}^{2+}$ concentration (Fig. 4) [32]. Recent studies suggest that the use of ultrasound plus microbubbles promotes intracellular increase of $\mathrm{Ca}^{2+}$ (a well-known regulator of the cytoskeleton), either by extracellular $\mathrm{Ca}^{2+}$ entry or release from the internal store without cellular membrane disruption [39]. This mechanism is highly specific since the polarisation is not modified in the absence of bubble oscillation or when the bubble is not in close contact with the membrane. This hyperpolarisation should facilitate, secondarily, molecular uptake through endocytosis and macropinocytosis [32].

\section{Results}

The literature contains a wealth of papers reporting positive results of sonoporation for both drug treatment and gene delivery. Since it is impossible to extensively report all the available results here, we propose a classification of the most attractive ones, based on clinical or pre-clinical evidence of sonoporation efficacy in treatment modalities. Among the different approaches investigated to exploit the combination of microbubbles and ultrasound for therapeutic purposes, sonothrombolysis has been investigated in great detail, yielding clinical evidence of clot dissolution.

\section{Sonothrombolysis/sonolysis}

Even though this is not the first area to be investigated, there is now clear evidence that the use of ultrasound and microbubbles can provide a clear improvement in animal models of stroke or heart infarct. Several pre-clinical studies have shown that the use of ultrasound plus 
microbubbles, with and without fibrinolytic drugs, is effective in improving recanalisation rates $[9,30,36]$. Similarly, for many years clinical trials conducted with or without a fibrinolytic agent, i.e. recombinant tissue plasminogen activator (r-tPa), have shown consistent improvements in recanalisation rates and clinical outcome, even though the latter has not been assessed systematically at 3 months in all these studies. Even though microbubbles plus ultrasound could be considered an effective tool for clot dissolution, in most of the cases r-tPa was associated as a free drug [30]. The underlying mechanism is linked to an improvement in the accessibility to fibrin strands by r-tPa, leading to an accelerated dissolution of the clot with a secondary recanalisation and rapid blood flow restoration in the area submitted to ischaemia. The presence of secondary adverse events reported in some clinical studies might not be related to the sonoporation itself, but more likely to the use of inadequate US settings or to a delay in treatment in relation to stroke onset.

\section{Oncology}

Unlike the situation with sonothrombolysis, where clinical trials have been conducted even without any approved indication for the use of microbubbles in this specific clinical situation, many pre-clinical studies have been conducted in various animal models for oncology applications [15, 22]. Most were conducted with doxorubicin, as this drug is suitable for use in many animal models, and considered size reduction or even disappearance of the tumour as the end point, even though this is probably not the most accurate or the earliest end point that could be used. However, almost all these studies reported a clear superiority of the association of US and non-encapsulated drug for size reduction. Moreover, this is clearly influenced by the selected acoustic parameters in most of the cases. Only very rarely was the drug encapsulated in the microbubbles. Few papers have dealt with other drugs, although one paper recently reported a convincing result with irinothecan in a rat model [14]. This confirms the great value of the proposed approach in oncology. The absence of clinical papers and, to the best of our knowledge, of ongoing clinical trials in this field is to be noted.

\section{Blood-brain barrier opening}

One of the most striking findings reported in recent years is that of the transitory opening of BBB under specific conditions. High mechanical pulses through intact skull, together with microbubbles flowing in vessels, are used in obtain this [2]. The local effect has been demonstrated by the local extravasation of Evan's Blue dye or by gadolinium extravasation on MRI immediately after the session. a
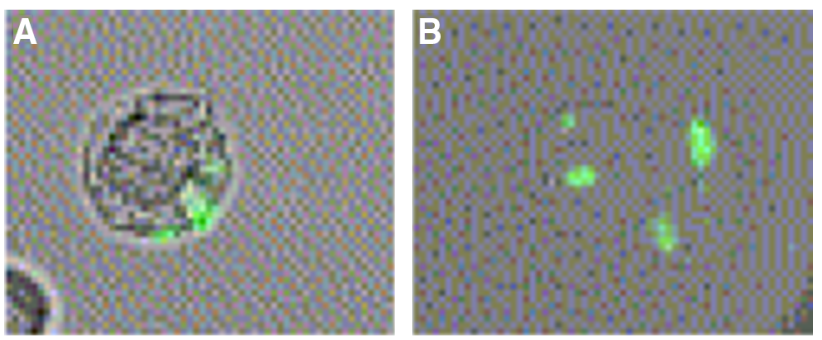

b
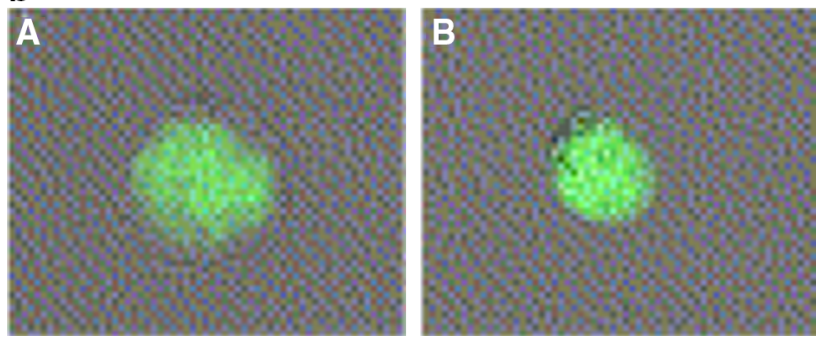

Fig. 5 a Lipid-mediated plasmid internalisation. MAT B III cells were incubated with LF 2000/(DNA/YOYO-1) lipoplexes and observed at $(A) 5 \mathrm{~min}$ and $(B) 1 \mathrm{~h}$ with a fluorescent microscope. The pictures correspond to the overlay of light transmission and epifluorescence images (using the analySIS software). Exposure time for the fluorescence microscopy observations: $100 \mathrm{~ms}$. Obj. $\times 100$. b Ultrasound-mediated plasmid internalisation. MAT B III cells were sonoporated with YOYO-1 labelled DNA and observed at (A) $5 \mathrm{~min}$ and $(B) 1 \mathrm{~h}$ post-sonoporation, using a focused transducer $(2.25 \mathrm{MHz})$ at $P=570 \mathrm{kPa}$ in the presence of a UCA at a concentration of 25 particles/cell (using the analySIS software). Exposure time for the fluorescence microscopy observations: $500 \mathrm{~ms}$. Obj. $\times 100$. Note that all the observed cells showed similar patterns of fluorescence [24] (color figure online)

One of the main advantages of this effect is, in fact, its very precise localisation, which is due to the fact that it is triggered by high-intensity focused ultrasound (HIFU), i.e. by ultrasound waves that are highly focused to deliver the required energy. This could open the way for treatment of brain diseases which, due to the presence of the $\mathrm{BBB}$, are not accessible to drugs [18, 24]. In addition, because the opening of the BBB is transient, the therapeutic approach is relatively safe for the patient. However, long-term studies must be conducted to confirm the absence of adverse events, since opening of the BBB, even transient, could allow the passage of other molecules or viruses and, potentially, result in irreversible lesions.

\section{Gene delivery}

In addition to delivering drugs via the mechanisms described and illustrated above, significant work has been done to increase the local delivery of plasmid DNA (pDNA) products. Indeed, although the final objectives are the same, the rationale differs significantly from the one adopted for drug release. Alternative methods have been 
proposed and are currently used to transfect various cell types. These include electroporation and non-viral or viral delivery methods. Even though the latter is recognised as very effective, viruses present various limitations. They are not effective on all cell types and in some cases accompanied by a certain degree of cell death, both limitations that are incompatible with their extensive use. Moreover, viruses could be considered problematic for clinical use due to the inherent associated risk. Therefore, knowing that gene therapy represents a great opportunity for some deleterious diseases, ultrasound-mediated transfection has been identified as one of the most promising fields, not only for demonstrating the efficacy of this approach but also because it is safe and easy to use in patients (Fig. 5) [4, 7, $10,13,15,16,21,25]$.

The best means of administering pDNA is still a matter of debate, as local or systemic administration can be used. To date, most of the published studies have been conducted with local administration of plasmids and in free form [23]. This is because of the inherent instability of plasmids in the blood stream resulting from DNase activity. Consequently, the relatively fast disappearance of plasmids after systemic injection means that a high dose is needed to ensure some efficacy; with local injection, on the other hand, lower doses should be sufficient. However, local administration cannot always be performed for human use. At present, both approaches are considered according to the selected organ of interest. Additional methods might be considered with the objective of protecting the plasmid from any rapid enzymatic degradation, the local delivery being obtained by applying ultrasound at the site of interest.

The local delivery mediated by combining ultrasound and microbubbles does not guarantee therapeutic efficacy, as there are additional points that must be carefully considered, such as the delivery of the plasmid to the right intracellular location and particularly to the nucleus. Therefore, ultrasound and microbubbles could greatly improve local delivery, but we cannot ignore the other factors involved in gene expression. Recent studies have investigated the use of small interfering RNA (siRNA) as a means of circumventing the problem of nucleus delivery. Indeed, this material, to be translated and become active, must be delivered only in the cytoplasm. This new approach has attracted some interest and could possibly become an important area in the future of this technology.

With regard to drug delivery, many diseases have been considered. The most active areas of research are those of cardiovascular disease and oncology, due to the vascular nature of the microbubbles used in this process.

In cardiovascular disease, the main objective is to facilitate or improve the myocardial remodelling after a myocardial infarct [16]. Indeed, due to the death of the myocardial cells during ischaemia, the affected area is subject to abnormal function and could thus expose the patients to deleterious consequences. The technique has been proposed as a means of restoring, at least partially, some cardiac function in this territory. One of the most attractive approaches is to restore blood flow in this area by stimulating neovascularisation, which is a normal process in healing. To this end, some teams have attempted to transfect the cells with angiogenic genes. One of the most common is the vascular endothelial growth factor $(V E G F)$ gene, and it has given positive results in terms of VEGF expression and secondary angiogenesis [21]. Similar approaches have been explored for hindlimb ischaemia, in order to restore a good balance between oxygen demand and local blood flow and therefore limit the clinical symptoms.

Another area of interest is the intimal hyperplasia observed in the course of stent insertion. Even though many techniques are currently used, from simple drugs to pre-conditioning of the stents, sonoporation could offer a valuable alternative by selectively inhibiting this intimal hyperplasia.

As described above in relation to drug delivery, oncology is an active area of research since this therapy could be of great value for lesions found to be resistant to conventional drugs $[4,15]$. Since some tumour lesions are not easily accessible, the main limitation of this approach is related to the fact that local delivery under ultrasound activation may not be optimal. To some extent this problem is similar to the one encountered for brain lesions for which HIFU is now proposed.

\section{Conclusion}

Gas microbubbles, used together with ultrasound waves, offer a unique opportunity to improve and control local drug/gene delivery. There is a body of evidence showing that this approach is very effective in vitro as well as in animal models. However, the situation of sonothrombolysis, for which clinical data are already available and demonstrate improved recanalisation rates, differs significantly from that of other drugs and gene delivery, where there is still lack of clinical data. It is wise to point out that precise control of each component is mandatory, since this will influence significantly both the efficacy and the safety of this method which must be non-invasive by essence. The underlying mechanisms have not been fully elucidated, but a close interaction between the cells and the microbubbles under acoustic activation is required. The specific nature of the microbubbles makes them suitable for imaging the lesions of interest and for local delivery under ultrasound activation, making this combination one of the most promising for theranostics. 
The recent progress made in this field, which has seen the emergence of convincing pre-clinical results, together with access to specific tissues, such as brain tissue, will likely change patient management in short term, allowing a significant reduction of adverse events and opening up opportunities that are still out of reach at the present time.

Conflict of interest François Tranquart, Thierry Bettinger and Jean Marc Hyvelin are Bracco employees.

Ethical standard This article does not contain any studies with human or animal subjects performed by any of the authors.

\section{References}

1. Alter J, Sennoga CA, Lopes DM, Eckersley RJ, Wells DJ (2009) Microbubble stability is a major determinant of the efficiency of ultrasound and microbubble mediated in vivo gene transfer. Ultrasound Med Biol 35:976-984

2. Beccaria K, Canney M, Goldwirt L, Fernandez C, Adam C, Piquet J, Autret G, Clément O, Lafon C, Chapelon JY, Carpentier A (2013) Opening of the blood-brain barrier with an unfocused ultrasound device in rabbits. J Neurosurg 119:887-898

3. Bohmer MR, Chlon CH, Raju BI, Chin CT, Shevchenko T, Klibanov AL (2010) Focused ultrasound and microbubbles for enhanced extravasation. J Control Release 148:18-24

4. Carson AR, McTiernan CF, Lavery L, Hodnick A, Grata M, Leng $X$ et al (2011) Gene therapy of carcinoma using ultrasound-targeted microbubble destruction. Ultra Med Biol 37:393-402

5. Castle J, Butts M, Healey A, Kent K, Marino M, Feinstein SB (2013) Ultrasound-mediated targeted drug delivery: recent success and remaining challenges. Am J Physiol Heart Circ Physiol 304:H350-H357

6. Choi JJ, Coussios CC (2012) Spatiotemporal evolution of cavitation dynamics exhibited by flowing microbubbles during ultrasound exposure. J Acoust Soc Am 132(5):3538-3549

7. Christiansen JP, French BA, Klibanov AL, Kaul S, Lindner JR (2003) Targeted tissue transfection with ultrasound destruction of plasmid bearing cationic microbubbles. Ultra Med Biol 29:1759-1767

8. Claudon M, Dietrich CF, Choi BI, Cosgrove DO et al (2013) Guidelines and good clinical practice recommendations for contrast enhanced ultrasound (CEUS) in the liver-update 2012: a WFUMB-EFSUMB initiative in cooperation with representatives of AFSUMB, AIUM, ASUM, FLAUS and ICUS. Ultraschall Med 34(1):11-29

9. Culp WC, Flores R, Brown AT, Lowery JD, Roberson PK, Hennings LJ, Woods SD, Hatton JH, Culp BC, Skinner RD, Borrelli MJ (2011) Successful microbubble sonothrombolysis without tissue-type plasminogen activator in a rabbit model of acute ischemic stroke. Stroke 42(8):2280-2285

10. Delalande A, Bouakaz A, Renault G, Tabareau F, Kotopoulis S, Midoux P et al (2011) Ultrasound and microbubble-assisted gene delivery in Achilles tendons: long lasting gene expression and restoration of fibromodulin KO phenotype. J Control Release 156: 223-230

11. de Jong N, Bouakaz A, Frinking P (2002) Basic acoustic properties of microbubbles. Echocardiography 19:229-240

12. Doinikov AA, Bouakaz A (2010) Acoustic microstreaming around an encapsulated particle. J Acoust Soc Am 127:1218-1227

13. Escoffre JM, Zeghimi A, Novell A, Bouakaz A (2013) In-vivo gene delivery by sonoporation: recent progress and prospects. Curr Gene Ther 13:2-14
14. Escoffre JM, Novell A, Serrière S, Lecomte T, Bouakaz A (2013) Irinotecan delivery by microbubble-assisted ultrasound: in vitro validation and a pilot preclinical study. Mol Pharm 10:2667-2675

15. Frenkel V (2008) Ultrasound mediated delivery of drugs and genes to solid tumors. Adv Drug Deliv Rev 60:1193-1208

16. Fujii H, Li SH, Wu J, Miyagi Y, Yau TM, Rakowski H et al (2011) Repeated and targeted transfer of angiogenic plasmids into the infarcted rat heart via ultrasound targeted microbubble destruction enhances cardiac repair. Eur Heart J 32:2075-2084

17. Geers B, Dewitte H, De Smedt SC, Lentacker I (2012) Crucial factors and emerging concepts in ultrasound-triggered drug delivery. J Control Release 164(3):248-255

18. Hynynen K, McDannold N, Vykhodtseva N, Jolesz FA (2001) Noninvasive MR imaging-guided focal opening of the bloodbrain barrier in rabbits. Radiology 220:640-646

19. Ibsen S, Schutt CE, Esener S (2013) Microbubble-mediated ultrasound therapy: a review of its potential in cancer treatment. Drug Design Dev Ther 3(7):375-388

20. Kooiman K, Emmer M, Foppen-Harteveld M, van Wamel A, de Jong $N$ (2010) Increasing the endothelial layer permeability through ultrasound activated microbubbles. IEEE Trans Biomed Eng 57:29-32

21. Leong-Poi H, Kuliszewski MA, Lekas M, Sibbald M, TeichertKuliszewska K, Klibanov AL et al (2007) Therapeutic arteriogenesis by ultrasound-mediated VEGF165 plasmid gene delivery to chronically ischemic skeletal muscle. Circ Res 101:295-303

22. Lentacker I, Geers B, Demeester J, De Smedt S, Sanders NN (2010) Design and evaluation of doxorubicin-containing microbubbles for ultrasound-triggered doxorubicin delivery: cytotoxicity and mechanisms involved. Mol Ther 18(1):101-108

23. Li YS, Davidson E, Reid CN, McHale AP (2009) Optimising ultrasound mediated gene transfer (sonoporation) in vitro and prolonged expression of a transgene in vivo: potential applications for gene therapy of cancer. Cancer Lett 273:62-69

24. Marty B, Larrat B, Van Landeghem M, Robic C, Robert P, Port M, Le Bihan D, Pernot M, Tanter M, Lethimonnier F, Mériaux S (2012) Dynamic study of blood-brain barrier closure after its disruption using ultrasound: a quantitative analysis. J Cereb Blood Flow Metab 32(10):1948-1958

25. Mehier-Humbert S, Bettinger T, Yan F, Guy RH (2005) Ultrasound mediated gene delivery: kinetics of plasmid internalization and gene expression. J Control Release 104:203-211

26. Mehier-Humbert S, Bettinger T, Yan F, Guy RH (2005) Plasma membrane poration induced by ultrasound exposure: implication for drug delivery. J Control Release 104:213-222

27. Meijering BD, Juffermans LJ, van Wamel A, Henning RH, Zuhorn IS, Emmer M, Versteilen AM, Paulus WJ, van Gilst WH, Kooiman K, de Jong N, Musters RJ, Deelman LE, Kamp O (2009) Ultrasound and microbubble-targeted delivery of macromolecules is regulated by induction of endocytosis and pore formation. Circ Res 104(5):679-687

28. Postema M, Marmottant P, Lancée CT, Hilgenfeldt S, de Jong N (2004) Ultrasound-induced microbubble coalescence. Ultrasound Med Biol 30(10):1337-1344

29. Rapoport N (2012) Phase-shift, stimuli-responsive perfluorocarbon nanodroplets for drug delivery to cancer. Interdiscip Rev Nanomed Nanobiotechnol 4(5):492-510

30. Ricci S, Dinia L, Del Sette M, Anzola P, Mazzoli T, Cenciarelli S, Gandolfo C (2012) Sonothrombolysis for acute ischaemic stroke. Cochrane Database Syst Rev 17:10

31. Tachibana K, Uchida T, Ogawa K, Yamashita N, Tamura K (1999) Induction of cell-membrane porosity by ultrasound. Lancet 353:1409

32. Tran TA, Roger S, Le Guennec JY, Tranquart F, Bouakaz A (2007) Effect of ultrasound-activated microbubbles on the cell electrophysiological properties. Ultrasound Med Biol 33:158-163 
33. Unnikrishnan S, Klibanov AL (2012) Microbubbles as ultrasound contrast agents for molecular imaging: preparation and application. Am J Roentgenol 199:292-299

34. van Wamel A, Bouakaz A, Versluis M, de Jong N (2004) Micromanipulation of endothelial cells: ultrasound-microbubblecell interaction. Ultrasound Med Biol 30:1255-1258

35. Wu J (1998) Temperature rise generated by ultrasound in the presence of contrast agent. Ultrasound Med Biol 24:267-274

36. Xie F, Slikkerveer J, Gao S, Lof J, Kamp O, Unger E, Radio S, Matsunaga T, Porter TR (2011) Coronary and microvascular thrombolysis with guided diagnostic ultrasound and microbubbles in acute ST segment elevation myocardial infarction. J Am Soc Echocardiogr 24(12):1400-1408
37. Yan F, Li X, Jin Q, Jiang C, Zhang Z, Ling T, Qiu B, Zheng H (2011) Therapeutic ultrasonic microbubbles carrying paclitaxel and lyp-1 peptide: preparation, characterization and application to ultrasound-assisted chemotherapy in breast cancer cells. Ultrasound Med Biol 37(5):768-779

38. Yudina A, Lepetit-Coiffé M, Moonen CT (2011) Evaluation of the temporal window for drug delivery following ultrasoundmediated membrane permeability enhancement. Mol Imaging Biol 13(2):239-249

39. Zhou Y, Shi J, Cui J, Deng CX (2008) Effects of extracellular calcium on cell membrane resealing in sonoporation. J Control Release 126:34-43 